An official journal of the / Un journal officiel de la "Société Sénégalaise de Cancérologie" (SOSECAN)

Journal homepage: www.africanjournalofoncology.com

Original article / Article original

DOI: https://www.doi.org/|0.54266/ajo.1.2.52.XRG|93|4

\title{
Management of cutaneous melanoma at the Joliot Curie Institute in Dakar
}

\section{Prise en charge des mélanomes cutanés à l'Institut Joliot Curie de Dakar}

JC. Balegana', S. Ka', D. Diouf', J. Thiam', M. Dieng', PM. Gaye', M. Diop', A. Dem'.

${ }^{1}$ Institut Joliot Curie de Dakar, Sénégal.

\begin{abstract}
AIM: To study the clinical and therapeutic aspects of cutaneous melanoma at the Joliot Curie Institute of Dakar. PATIENTS AND METHODS: We performed a retrospective, descriptive and critical study at the Joliot Curie Institute of Dakar including all histologically confirmed melanoma cases between January 2008 and December 2013. RESULTS: During the study period, 21 cases were managed. The location was plantar in $76 \%$ of cases. Acral type was found in $85.7 \%$ of cases. All patients had a Clark level of IV or higher with a Breslow index of more than two mm. Fourteen patients underwent excision surgery and 12 had inguinal lymph node dissection. The average follow-up was 16 months with an overall survival of $58.7 \%$ at six months and $51 \%$ at one year. CONCLUSION: The majority of patients have benefited from surgical treatment, without prescription of immunotherapy which to date has revolutionized the management of advanced melanoma.

KEYWORDS: Melanoma; Joliot Curie Institute; Dakar.
\end{abstract}

BUT : Etudier les aspects cliniques et thérapeutiques des mélanomes cutanés à l'Institut Joliot Curie de Dakar. PATIENTS ET METHODES : Nous avons réalisé une étude rétrospective, descriptive et critique à l'Institut Joliot Curie de Dakar incluant tous les cas de mélanomes confirmés par l'histologie entre Janvier 2008 et Décembre 20I3. RESULTATS : Au cours de la période d'étude, 21 cas ont été pris en charge. La localisation était plantaire dans $76 \%$ des cas. Le type acral était retrouvé dans $85,7 \%$ des cas. Tous les patients avaient un niveau de Clark supérieur ou égal à IV avec un indice de Breslow supérieur à deux mm. La chirurgie d'exérèse était réalisée chez 14 patients avec curage ganglionnaire inguinale chez 12 patients. La moyenne de suivi était de 16 mois avec une survie globale de $58,7 \%$ à six mois et $51 \%$ à un an. CONCLUSION : La majorité des patients présentait un mélanome acral et a bénéficié d'un traitement chirurgical, aucune immunothérapie qui à ce jour a révolutionné la prise en charge des mélanomes avancés n'a été administrée.

MOTS-CLES : Mélanome ; Institut Joliot Curie ; Dakar.

\section{INTRODUCTION}

Le mélanome cutané est une prolifération maligne au dépend des mélanocytes cutanés situés dans la couche basale de l'épiderme. II est le cancer cutané le plus agressif avec un potentiel métastatique très élevé. Son incidence est en augmentation dans la population caucasienne avec près de 200.000 nouveaux cas chaque année dans le monde [I]. Les facteurs de risque chez les caucasiens sont bien connus. Chez le sujet noir, le mélanome reste rare et les facteurs de risque ne sont pas bien élucidés [2-4]. Certains sont suspectés notamment les traumatismes, la marche pied nu ou encore l'anomalie du gène MCRI. Le principal traitement reste la chirurgie qui permet une guérison pour les stades précoces. Le pronostic reste péjoratif pour les stades avancés et métastatiques. Actuellement avec le développement des thérapies cibles qui améliorent la survie des malades métastatiques mais avec une mortalité non négligeable $[5,6]$.

\section{PATIENTS ET METHODES}

C'était une étude rétrospective descriptive sur six ans qui couvrait la période de Janvier 2008 à Décembre 2013 et portait sur les dossiers des patients présentant un cancer cutané confirmé par l'histologie (biopsie ou pièce opératoire). Les données ont été analyses par Excel(C 2013 et le logiciel
STATA@ version 12. Pour comparer les données, nous avons utilisé le test statistique paramétrique de Chi deux de Pearson. Le seuil de significativité était fixé à $\mathrm{p}=0,05$. Les survies ont été calculées en utilisant la méthode de Kaplan-Meier. Le test LogRank a été utilisé pour comparer les courbes de survie en fonction des différents facteurs pronostiques étudies.

\section{RESULTATS}

Cent-et-trente-et-six cas des cancers cutanés étaient pris en charge dans l'institut dont 21 cas de mélanome retenus pour notre étude, soit $15,4 \%$ des tous les cancers cutanés. L'âge moyen de nos patients était de 60,8 ans avec des extrêmes de 29 ans et 85 ans. Le sex-ratio était de 0,75 . Le délai moyen de consultation de nos patients était de 32,8 mois avec les extrêmes de quatre et 108 mois. Tous nos patients étaient des sujets noirs. Aucun cas d'antécédent familial de mélanome n'a été retrouvé dans notre étude. La tumeur siégeait au niveau plantaire dans $76 \%$ des cas $(n=16)$ (Tableau I). La taille tumorale moyenne était de $7,1 \mathrm{~cm}$ avec des extrêmes de 2,5 et $15 \mathrm{~cm}$. La présence d'ulcération tumorale a été retrouvée chez 15 patients soit $71 \%$ des cas. L'absence de signe d'envahissement ganglionnaire régional était retrouvée chez huit patients soit $38 \%$ des cas. Des ganglions inguinaux étaient retrouvés chez II patients soit 52,4\%. Le mélanome acral 
lentigineux était le type histologique le plus retrouvé chez 18 patients soit $\mathbf{8 5 , 7 \%}$ des cas (Tableau II).

Tableau I : Répartition en nombre et pourcentage selon le siège de la tumeur.

\begin{tabular}{|l|l|l|}
\hline Siège & Nombre & Pourcentage (\%) \\
\hline Plante de pied & I6 & 76 \\
\hline Dorso-lombaire & I & 4,8 \\
\hline Paroi abdominale & I & 4,8 \\
\hline Paroi thoracique & I & 4,8 \\
\hline Hallux droit & I & 4,8 \\
\hline $3^{\text {ème }}$ orteil & I & 4,8 \\
\hline Total & 21 & 100 \\
\hline
\end{tabular}

Tableau II : Types histologiques.

\begin{tabular}{|lll|}
\hline Type histologique & Nombre & Pourcentage (\%) \\
\hline Acral & 18 & 85,7 \\
Nodulaire & 2 & 9,5 \\
SSM & 1 & 4,8 \\
Dubreuilh & 0 & 0 \\
\hline Total & 21 & 100 \\
\hline
\end{tabular}

Toutes les tumeurs présentaient un niveau de Clark supérieur IV. L'indice de Breslow était précisé chez six patients respectivement $2,2 \mathrm{~mm}$, quatre $\mathrm{mm}$, cinq $\mathrm{mm}$, huit $\mathrm{mm}, 10 \mathrm{~mm}$ et $30 \mathrm{~mm}$. Les métastases étaient retrouvées chez huit patients, soit quatre synchrones et quatre métachrones. La chirurgie était réalisée chez I4 patients, soit 66,7 \%. L'exérèse était réalisée chez huit patients avec des marges macroscopiques de trois $\mathrm{cm}$. L'amputation et/ou la désarticulation étaient réalisées chez six patients (Tableau III). Le curage ganglionnaire inguinal était réalisé chez 12 patients, soit $57,1 \%$ des cas.

Tableau III : Répartition selon le type de chirurgie.

\begin{tabular}{|c|c|c|}
\hline Type de chirurgie & Nombre & Pourcentage (\%) \\
\hline Exérèse tumorale & 8 & 57,14 \\
\hline Amputation de la jambe & I & 7,14 \\
\hline $\begin{array}{l}\text { Désarticulation de la } \\
\text { hanche }\end{array}$ & I & 7,14 \\
\hline $\begin{array}{l}\text { Désarticulation de } \\
\text { Chopart }\end{array}$ & I & 7,14 \\
\hline Désarticulation d'orteil & 3 & 21,43 \\
\hline Total & 14 & 100 \\
\hline
\end{tabular}

L'examen histologique des pièces opératoires avait découvert une prédominance de type acral lentigineux chez $57,1 \%(n=8)$. Les marges d'exérèse étaient saines chez huit patients, soit $57 \%$ des cas. Par contre, les marges d'exérèse chirurgicale n'ont pas été précisées chez six patients, soit $43 \%$ des cas. Le nombre moyen de ganglion retrouvé dans le produit de curage était de sept ganglions avec un envahissement dans 4I,7\% (cinq cas). Les complications liées à la chirurgie retrouvées dans notre étude étaient la suppuration locale et le lâchage de suture chez quatre patients, soit $28,6 \%$ des cas. La chimiothérapie était réalisée chez 5 (23,8\% des cas) dont adjuvant chez trois patients et à visée palliative chez deux patients avec comme molécule de base la dacarbazine. La radiothérapie était réalisée chez un patient à visée hémostatique. Pour un suivi moyen de 16 , I mois, la survie globale était de $58,8 \%$ à six mois, de $51,5 \%$ à 12 mois (Figure I).

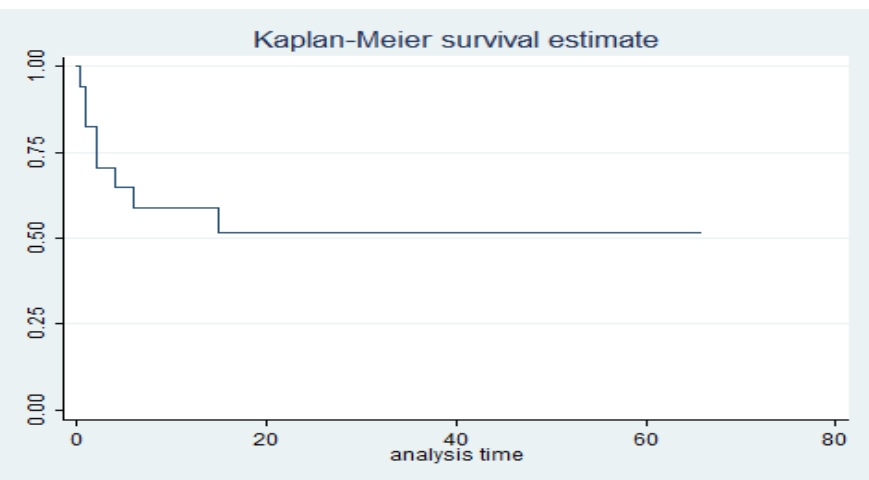

Figure I : Courbe de la survie globale.

Les patients qui ne présentaient pas d'adénopathies semblaient avoir une meilleure survie par rapport à ceux qui avaient des adénopathies (Figure 2).

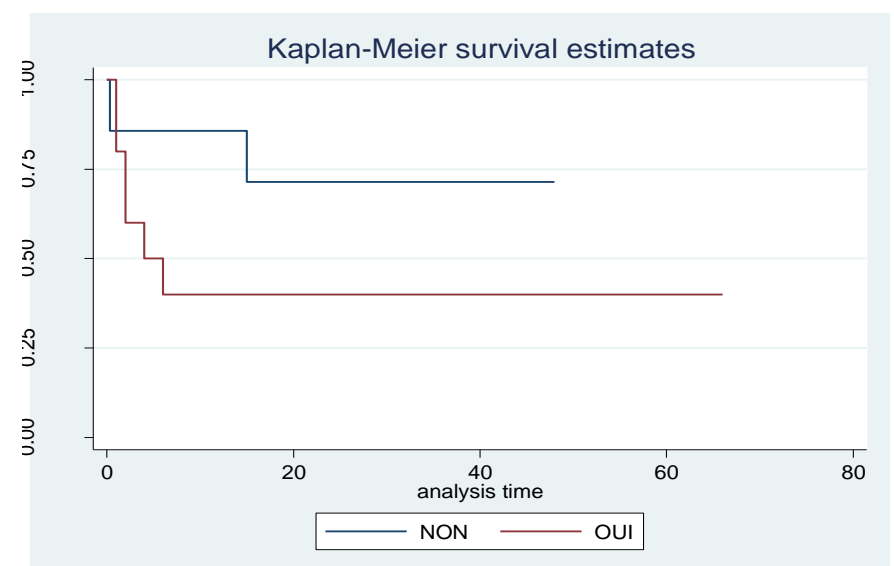

Figure 2 : Comparaison des courbes de survie selon la présence ou l'absence des adénopathies $(\mathrm{Chi} 2(\mathrm{I})=\mathrm{I} .8 \mathrm{I}, \mathrm{Pr}>\mathrm{Chi} 2=0.1783)$.

\section{DISCUSSION}

Dans notre série, les mélanomes représentaient 15,4\% des tous les cancers cutanés avec un sex-ratio de 0,75 . Pitche et al [2] retrouvaient seulement quatre cas de mélanomes soit $5,8 \%$ de même que Saka et al [3] notaient 23 cas de mélanomes soit $10,3 \%$ des cancers cutanés avec un sex-ratio de 0,6 en dermatologie au Togo. Ces différentes études menées en Afrique Noire montrent que le mélanome demeure un cancer rare chez le sujet noir par rapport à la population blanche dans laquelle la fréquence de ce cancer est en augmentation [7, 8]. Cinquante-sept virgule un pour cent de nos patients étaient âgés de plus de 60 ans.

Dans notre travail, la tumeur siégeait au niveau des membres inférieurs (MI) notamment plantaire, dans $85,7 \%$ des cas. Cette situation est observée dans quasiment toutes les séries africaines comme celle de Tarwate et al au Maroc [9], Soua et al en Tunisie [10], Amalia et al au Nigéria [4] ou dans celle de Mohamed et al à Jos au Nigéria [I l].

L'ulcération tumorale, un des facteurs pronostiques du mélanome, était retrouvée dans notre étude dans $71 \%$ des cas 
confirmant un stade évolué et agressif chez la majorité de nos patients. Cette situation peut s'expliquer par un retard de consultation du fait de la localisation plantaire de la tumeur qui est souvent méconnue et négligée par les patients d'un âge avancé.

Dans notre série, le type acral était le plus retrouvé dans $85,7 \%$ ce qui corrobore avec la majorité des séries chez le sujet noir. Contrairement chez le caucasien, le SSM est le type histologique le plus fréquent $[8,12]$.

Tous nos patients présentaient des tumeurs ayant un niveau de Clark supérieur ou égale à IV ce qui confirme le caractère très évolué de la maladie dans notre série.

La chirurgie d'exérèse tumorale était réalisée chez $66,1 \%$ de nos patients et associée à un curage inguinal dans $57,1 \%$ des cas. Ceci implique qu'environ $40 \%$ des patients n'ont pas subi la chirurgie, soit du fait de leur maladie métastatique dans $19 \%$ des cas, soit du fait de la non adhésion au traitement ou encore un problème purement financier. Dans notre étude, nous n'avons pas réalisé de ganglion sentinelle (GS), le curage inguinal était systématique d'autant plus que $52,4 \%$ des patients présentaient des adénopathies inguinales. Cette technique est préconisée pour les tumeurs de plus d'un $\mathrm{mm}$ d'épaisseur en se basant sur les résultats de Morton et al sur le GS que la survie était de $90 \%$ à cinq ans en l'absence de métastase ganglionnaire et de $72,3 \%$ à cinq ans s'il existait des métastases ganglionnaires [13].

Seulement $24 \%$ des patients avaient reçus une chimiothérapie à base de dacarbazine. Aucun de nos patients n'a reçu une immunothérapie ou thérapie ciblée en adjuvant ou en situation métastatique. Cette attitude s'explique du fait de la difficulté d'accès à ces molécules qui coûtent extrêmement chers et sont non remboursables. Dans la recommandation actuelle, l'immunothérapie et la thérapie ciblée notamment les inhibiteurs de BRAFV600E (Vemurafénib®) sont devenus une référence dans la prise en charge des mélanomes avancés et métastatiques. Les perspectives thérapeutiques pour les mélanomes métastatiques seront de combiner les inhibiteurs de BRAF et de MEK avec les résultats supérieurs par rapport aux inhibiteurs de BRAF seul. Plusieurs essais sont en cours avec des résultats encourageants [14]. Actuellement, l'immunothérapie avec les inhibiteurs de check point est devenue un standard dans la prise en charge en première ligne de mélanome métastatique dont notamment les anti PD-I qui ont montré leur efficacité en termes de survie sans progression et survie globale par rapport à l'ipilimumab [15].

\section{CONCLUSION}

Le mélanome cutané sur peau noire est rare mais non exceptionnel avec une agressivité importante et un pouvoir métastatique élevé. La localisation est souvent plantaire méconnu expliquant une consultation tardive avec des tumeurs évoluées voir métastatiques ce qui rend le pronostic péjoratif. L'immunothérapie par les inhibiteurs de check point doit rentrer dans nos pratiques quotidiennes pour les cas avancés et métastasiques. II faut sensibiliser la population sur l'intérêt d'une consultation précoce et toute lésion plantaire suspect doit être excisé en totalité pour un examen histologique.

\section{REFERENCES}

I. Globocan 2012.

2. Pitche P, Tchamdja S, Napo koura G, Bakonde P, Kpodzro K, Tchangaï-Walla K.Les cancers cutanés en consultation dermatologique à Lomé (Togo). Médecine d'Afrique Noire : 1997, 44 (I) :I5-I7.

3. Saka B , Souley Z, Kombaté K, Mouhari-Toure A, Akakpo S, Napo-Koura $G$ et al. Les cancers cutanés au Togo : 223 observations. Med Trop 2010 ; 70(2) : |69-17|.

4. Samalia MAO, Rafindadi AH.Pattern of cutaneous malignant melanoma in Zaria.Nigeria.Annals of African Medecine Society 2006; 5(I):16-19.

5. Graham G. Giles, Bruce K Armstrong, Robert C Burton, Margaret P. Staples, Vicky J. Thursfield.Has mortality from melanoma stopped rising in Australia? Analysis of trends between 193I and 1994,BMJ 1996; 312(7039): I I 2 |- I I 25.

6. Maraninchi $D$, Cerf $N$, Bousquet $P$.Dynamique d'évolution des taux de mortalité des principaux cancers en France, Institut national du cancer. France, Paris Nov 201 I:47-48.

7. Bulliard J, Panizzon R, Levi F.Epidémiologie et prévention du mélanome cutané en suisse.forum Med Suisse 2009, 9(17) :3।4.

8. Mackie RM, Bray C, Vestey J, Doherty V, Evans A, Thomson $D$ et al. Melanoma incidence and mortality in Scotland 1979-2003, British Journal of Cancer (2007);96, 1772 - 1777.

9. Tarwate $M$, Benchikhi $H$, Adarmouch $L$, Benider $A$, Amine $M$, Zamiati $S$. Mélanome épais facteurs de mortalité et de survenu des métastases. Pan African Medical Journal 2014; I8(44) :28I2.

10. Soua Y, Chaabane H, Meziou T, Khemakhem H, Mseddi M.Profil anatomoclinique du melanome dans le sud tunisien Ann derm. 201 I.10.384.

II. Mohammed A, Manasseh A, Mandong B, Edino S.Histopathological study of malignant melanoma in highlanders. Nigerian Journal of Surgical Research 2003; 5:18-22.

12. Howlett A, Dewar R, Morris S.The epidemiology of cutaneous malignant melanoma in Nova Scotia.Can J Plast Surg 2006; I4 (4) : 21 I-2। 4.

13. Morton DL, Thompson JF, Cochran AJ, Mozzillo N, Elashoff R, Essner $R$ et al. Sentinel node biopsy or nodal observation in melanoma. N Engl J Med 2006 ; 355(I3) : I307-1317.

14. Long GJ, Stroyakovskiy D, Gogas H, Levchenko E, Braud F, Larkin J et al.Combined BRAF and MEK Inhibition versus BRAF Inhibition Alone in Melanoma. N Engl J Med 20|4; 37I (20): I877-I888.

15. Carollina R, Georgina V, schachter J. Long-term outcomes in patients (pts) with ipilimumab (ipi)-naive advanced melanoma in the phase 3 KEYNOTE-006 study who completed pembrolizumab (pembro) treatment. J Clinical oncology 2017 ; 35(15)95049504.

\section{CONFLITS D'INTERET}

Les auteurs n'ont déclaré aucun conflit d'intérêts. 